\title{
Differential Stability of PNS and CNS Nodal Complexes When Neuronal Neurofascin Is Lost
}

\author{
Anne Desmazieres, ${ }^{\star}$ Barbara Zonta, ${ }^{\star}$ Ao Zhang, Lai-Man N. Wu, Diane L. Sherman, and Peter J. Brophy \\ Centre for Neuroregeneration, University of Edinburgh, Edinburgh EH16 4SB, United Kingdom
}

Fast, saltatory conduction in myelinated nerves requires the clustering of voltage-gated sodium channels (Nav) at nodes of Ranvier in a nodal complex. The Neurofascin ( Nfasc) gene encodes neuronal Neurofascin 186 (Nfasc186) at the node and glial Neurofascin 155 at the paranode, and these proteins play a key role in node assembly. However, their role in the maintenance and stability of the node is less well understood. Here we show that by inducible ablation of Nfasc in neurons in adult mice, Nfasc186 expression is reduced by $>99 \%$ and $94 \%$ at PNS and CNS nodes, respectively. Gliomedin and NrCAM at PNS and brevican at CNS nodes are largely lost with neuronal neurofascin; however, Nav at nodes of Ranvier persist, albeit with $\sim 40 \%$ reduction in expression levels. $\beta$ IV Spectrin, ankyrin $\mathrm{G}$, and, to a lesser extent, the $\beta 1$ subunit of the sodium channel, are less affected at the PNS node than in the CNS. Nevertheless, there is a $38 \%$ reduction in PNS conduction velocity. Loss of Nfasc186 provokes CNS paranodal disorganization, but this does not contribute to loss of Nav. These results show that Nav at PNS nodes are still maintained in a nodal complex when neuronal neurofascin is depleted, whereas the retention of nodal Nav in the CNS, despite more extensive dissolution of the complex, suggests a supportive role for the partially disrupted paranodal axoglial junction in selectively maintaining Nav at the CNS node.

Key words: neurofascin; nodes of Ranvier; sodium channels; myelination

\section{Introduction}

Myelinated axons conduct impulses rapidly because the $\alpha$ subunits of voltage-gated sodium channels (Nav) and their accessory proteins become clustered at nodes of Ranvier. In addition to voltage-gated sodium channels, the nodal complex includes $\beta$ IV spectrin, ankyrin G, a neuronal isoform of neurofascin, Neurofascin 186 (Nfasc186), contactin (in the CNS), NrCAM, and gliomedin (in the PNS); Davis et al., 1996; Tait et al., 2000; Jenkins and Bennett, 2002; Yang et al., 2004; Eshed et al., 2005). The paranodal axoglial junctions flank the nodes in an adhesion complex comprising the glial isoform of neurofascin, Neurofascin 155 (Nfasc155), and the axonal proteins Caspr (also known as paranodin) and contactin (Menegoz et al., 1997; Tait et al., 2000;

Received Nov. 4, 2013; revised Dec. 26, 2013; accepted Dec. 31, 2013.

Author contributions:A.D., B.Z., and P.J.B. designed research; A.D., B.Z., A.Z., and L.-M.N.W. performed research; D.L.S. contributed unpublished reagents/analytic tools; A.D., B.Z., and P.J.B. analyzed data;A.D. and P.J.B. wrote the paper.

Author contributions: A.D., B.Z., and P.J.B. designed research; A.D., B.Z., L.-M.N.W., and A.Z. performed research; A.D., B.Z., D.L.S., and P.J.B. analyzed data; D.L.S. contributed an essential reagent; P.J.B. and A.D. wrote the paper. This study was supported by the Wellcome Trust. We thank Heather Anderson and Stuart Fleming for excellent technical assistance.

${ }^{*}$ A.D. and B.Z contributed equally to this work.

The authors declare no competing financial interests.

Correspondence should be addressed to Peter J. Brophy, Centre for Neuroregeneration, University of Edinburgh, Chancellor's Building, 49 Little France Crescent, Edinburgh, EH16 4SB, UK. E-mail: Peter.Brophy@ed.ac.uk.

A. Desmazieres' present address: CRICM INSERM-UPMC UMRS 975, CNRS 7225, Hôpital de la Salpêtrière, 75651 Paris Cedex 13, France.

B. Zonta's present address: Department of Pharmacology, Oxford University, Oxford, OX1 30T, UK.

L.-M.N. Wu's present address: Department of Developmental Biology, University of Texas Southwestern Medical Center, Dallas, TX 75390.

DOI:10.1523/JNEUROSCI.4662-13.2014

Copyright $\odot 2014$ the authors $\quad 0270-6474 / 14 / 345083-06 \$ 15.00 / 0$
Bhat et al., 2001; Boyle et al., 2001; Charles et al., 2002; Sherman et al., 2005).

The Neurofascin (Nfasc) gene is uniquely essential for the assembly of the nodal complex (Sherman et al., 2005). Nfasc encodes a neuronal isoform, Nfasc186, and a glial isoform, Nfasc155, by alternative splicing (Davis et al., 1996; Tait et al., 2000). Nfasc186 has a key role in clustering sodium channels (Zonta et al., 2008; Feinberg et al., 2010; Thaxton et al., 2011). The paranodal axoglial junctional complex can independently cluster Nav at nodes during development (Zonta et al., 2008; Feinberg et al., 2010), although a recent paper has disputed this (Thaxton et al., 2011).

Here, we show that there are significant differences between CNS and PNS nodal complexes in their susceptibility to disruption after the elimination of nodal neurofascin in the adult mouse. Nevertheless, in both cases, Nav are notably resistant to loss. We attribute this partly to the continued presence of intact paranodal junctions. However, age-related partial mislocalization of junctional proteins at CNS paranodes, exacerbated by the loss of nodal neurofascin, did not accelerate the decline in Nav maintenance at the node. We suggest that Nav are maintained at nodes through a combination of their interactions with nodal components in addition to neuronal neurofascin, together with restrictions on their diffusion caused by the paranodal adhesive junction, with the latter being particularly important in the CNS. This provides further evidence that the mechanisms of nodal stabilization depend on protein-protein interactions that are different from those that dominate initial assembly, as has been demonstrated for Nav at the axon initial segment (AIS; Zonta et al., 2011). 

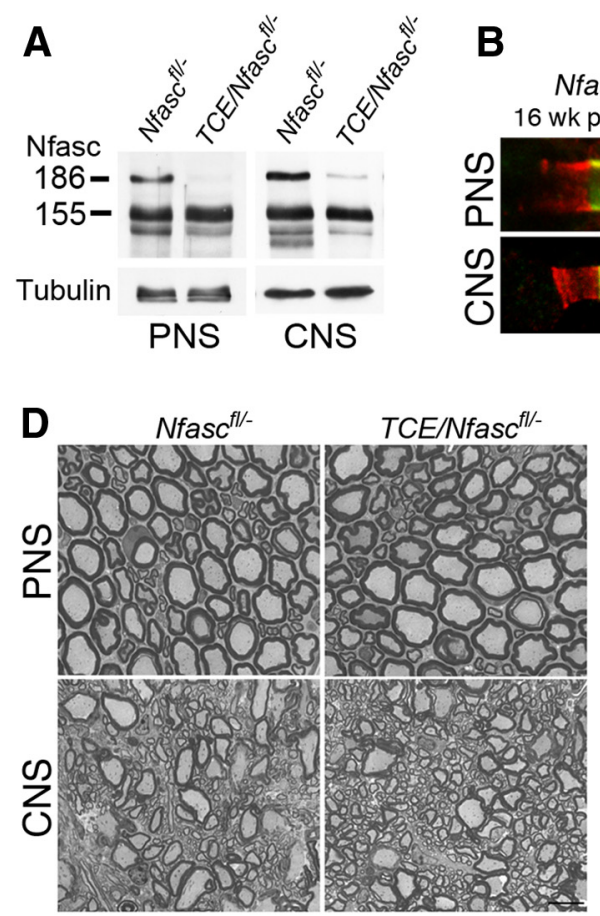

B

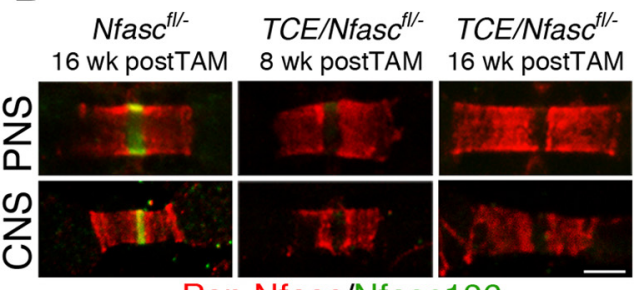

Pan Nfasc/Nfasc186

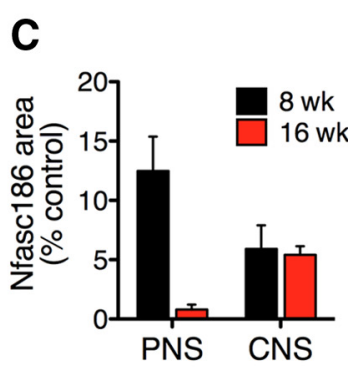

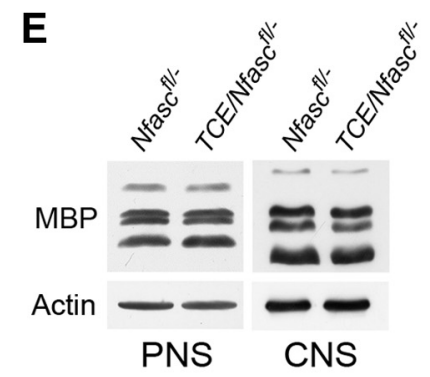

Figure 1. Characterization of Nfasc 186 deletion and preservation of myelin in the TCE/Nfass ${ }^{f / /}$ model. Control (Nfass ${ }^{f / /}$ ) and mutant (TCE/Nfast $\mathrm{fl}^{f /-}$ ) mice received intraperitoneal injections of tamoxifen to induce the recombination of the Nfasc floxed allele. $A$, Western blotting of peripheral nerve and spinal cord lysates from control and mutant mice 16 weeks post-tamoxifen shows substantial loss of Nfasc186, while glial Nfasc 155 is unaffected. Tubulin was the loading control. B, Immunofluorescence of teased quadriceps nerves (PNS) and ventral funiculi of cervical spinal cord (CNS) from Nfase $C^{f /-}$ and TCE/Nfase $C^{f /-}$ mice shows the disappearance of nodal Nfasc 186 at 8 weeks (wk) and 16 weeks post-tamoxifen in both PNS and CNS. The Pan Nfasc antibody recognizes both Nfasc 186 and Nfasc 155 . Scale bar, $3 \mu \mathrm{m}$. C, Quantitation of nodal area immunoreactive for Nfasc 186 in mutant quadriceps nerves and ventral funiculi at 8 weeks and 16 weeks post-tamoxifen as

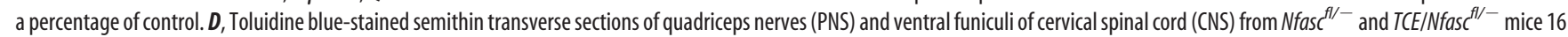
weeks post-tamoxifen show no obvious alteration of myelin in myelinated axons. Scale bar, $10 \mu \mathrm{m}$. E, Western blotting of peripheral nerve and spinal cord lysates 16 weeks post-tamoxifen shows normal amounts of the myelin basic proteins (MBP) in Nfast $\mathrm{fl}^{\mathrm{f} /}$ and TCE/Nfasc $\mathrm{fl}^{\mathrm{fl}-}$ mice. Actin was the loading control.

\section{Materials and Methods}

Animals. All animal work conformed to UK legislation (Scientific Procedures) Act 1986 and to the University of Edinburgh Ethical Review Committee policy, and mice were of either sex. Mice carrying alleles for $\mathrm{Nfasc}^{-}, \mathrm{Nfasc} \mathrm{fl}^{\mathrm{fl}}$, and the characterization of ThyCreERT2 (TCE) mice have been described (Sherman et al., 2005; Zonta et al., 2011). The TCE line was interbred with $\mathrm{Nfasc}^{+/-}$and $\mathrm{Nfasc} \mathrm{cllfl}^{\mathrm{fl}}$ mice to generate the TCE/ $\mathrm{Nfasc}^{-/ f l}$ mice used for the present study. All mice were on the C57BL/6 background, and the quadriceps nerve and the ventral funiculus of the spinal cord were the source of PNS and CNS tissue, respectively. The tamoxifen injection protocol has been described previously (Zonta et al., 2011).

Antibodies, microscopy, and Western blots. Most primary and secondary antibodies, together with methods for immunofluorescence, electron microscopy, and Western blotting, have been previously described (Tait et al., 2000; Sherman et al., 2005; Zonta et al., 2008, 2011; Sherman et al., 2012). We also used rabbit anti-Gliomedin (1:500; E. Peles, Weizmann Institute of Science, Rehovot, Israel), mouse anti-Kv1.1, IgG2b K36/15 (1:100) (NeuroMab, UC Davis/NIH NeuroMab Facility), and rabbit anti-brevican (1:500; C. Seidenbecher, C.I. Leibniz Institute for Neurobiology, Magdeburg, Germany). Rabbit anti- $\beta$ IV spectrin (1:100) and anti- $\beta 1$ sodium channel subunit (C terminus, $1: 100)$ were generated after immunization with the TDEGNPKREGGERRASGRRK and TSESKENCTGVQVAE synthetic peptides, respectively, each with an $\mathrm{N}$-terminal cysteine conjugated to KLH. Each antibody was affinitypurified by immunoabsorption to a column of peptide coupled to sulfoMBS-activated aminohexyl Sepharose 4B (Sigma). Samples were mounted in Vectashield (Vector Laboratories) and images were acquired with a confocal microscope (TCL-SL, Leica) using identical settings for control and mutant animals. For quantitation of stained nodal area, a series of four stacks per node of Ranvier was acquired under nonsaturating conditions. Nodal mean areas were obtained by averaging the values of the four corresponding stacks. For all nodal area measurements, at least 30 nodes were analyzed per animal, with at least 3 animals per genotype.

Statistical analyses were performed by either Student's $t$ test or oneway ANOVA followed by Tukey's multiple-comparison tests using GraphPad Prism 5.0c software. All figures were prepared using Adobe Photoshop CS4 extended version 11.

Nerve conduction velocity. Quadriceps nerves were transferred from oxygenated HEPES physiological solution (containing, in mM: $137 \mathrm{NaCl}$, $5 \mathrm{KCl}, 2 \mathrm{CaCl}_{2}, 1 \mathrm{MgCl}_{2}, 5.5$ D-glucose, and 5 HEPES, $\mathrm{pH}$ 7.2-7.4) to an isolated chamber containing an array of $\mathrm{Ag} / \mathrm{AgCl}$ electrodes at $1 \mathrm{~mm}$ intervals, surrounded by liquid paraffin, and maintained at $37^{\circ} \mathrm{C}$ no longer than $10 \mathrm{~min}$. Compound action potentials were recorded as described previously (Court et al., 2004). Recordings were repeated two to three times for each nerve. Conduction velocity was determined for each animal by averaging the mean conduction velocities from both nerves, and five animals were studied per genotype.

\section{Results}

Tamoxifen-induced loss of Nfasc186 at PNS and CNS nodes Cre-mediated inactivation of the floxed allele in TCE/Nfasc $\mathrm{fll}^{-}$ mice was initiated by intraperitoneal injection of tamoxifen, at the end of which the mice were 6 weeks old. Loss of neuronal neurofascin was assessed by Western blot (Fig. 1A) and by immunofluorescence (Fig. $1 B, C$ ). Loss of Nfasc186 was confirmed by Western blot at 16 weeks and by immunofluorescence at 8 and 16 weeks post-tamoxifen (Fig. 1A,B). The isoform of myelinforming glia, Nfasc155, was unaffected in both the CNS and PNS in the mutant (Fig. 1A). Quantitative immunofluorescence microscopy showed that loss of Nfasc186 at PNS nodes was essentially complete by 16 weeks post-tamoxifen $(>99 \%)$, whereas 
A
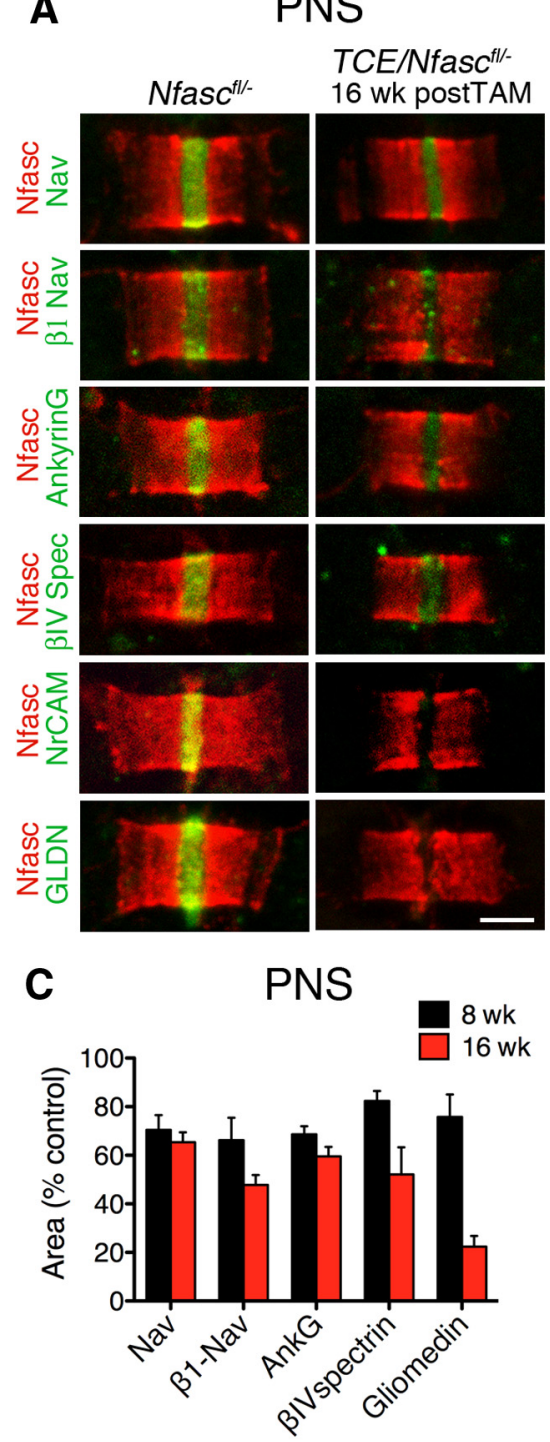

B
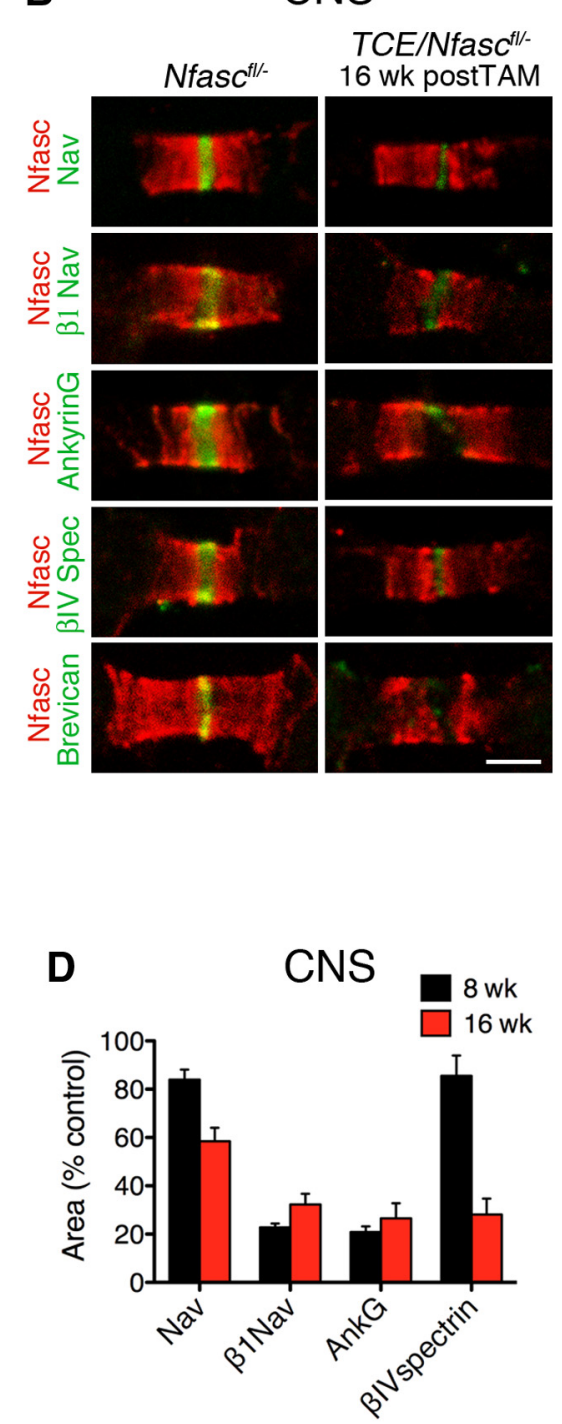

Figure 2. Loss of Nfasc186 leads to differential loss of nodal proteins in the PNS and CNS. $\boldsymbol{A}, \boldsymbol{B}$, Immunofluorescence of quadriceps nerves (PNS; $\boldsymbol{A}$ ) and spinal cord ventral funiculi (CNS; $\boldsymbol{B})$ from control (Nfasc $\mathrm{fl}^{\mathrm{fl}-}$ ) and mutant (TCE/Nfasc ${ }^{\mathrm{fl}-}$ ) ) mice at 16 weeks (wk) post-tamoxifen shows disruption of nodal components following inducible deletion of Nfasc186. $\beta$ IV Spec, $\beta$ IV Spectrin; GLDN, gliomedin. $\boldsymbol{C}, \boldsymbol{D}$, The quantitation of nodal disruption is shown for PNS $(\boldsymbol{C})$ and CNS (D) where mean nodal areas \pm SEM for the mutant are expressed as percentage of control mean nodal area at 8 and 16 weeks post-tamoxifen treatment. Scale bar, $3 \mu \mathrm{m}$.

$\sim 6 \%$ of CNS nodal Nfasc186 persisted. Interestingly, although ablation of neuronal neurofascin at PNS nodes was more complete than at CNS nodes, it seemed to proceed with different kinetics. At 8 weeks post-tamoxifen in the PNS, $\sim 88 \%$ of the Nfasc186 was lost, whereas at the same time point in the CNS, nodal neurofascin levels had reached those observed at 16 weeks. Nevertheless, and as we have observed previously, the slow loss of nodal neurofascin by comparison with Nfasc186 at the AIS indicates that this protein is remarkably stable in the nodal complex (Zonta et al., 2011).

Loss of neuronal neurofascin did not affect the myelin sheath. Electron microscopy of transverse sections of PNS and CNS nerves from $N f a s c^{f l l-}$ and TCE/Nfasc ${ }^{f l-}$ mice 16 weeks posttamoxifen did not reveal any obvious derangements to the sheath, and this was supported by Western blots of lysates from those nerves, which showed that myelin basic protein levels were normal in the mutants (Fig. $1 D, E$ ).

Loss of neuronal neurofascin causes selective loss of nodal components We assessed changes in immunofluorescence staining of nodal components by two methods: either we quantitated the area of staining under standardized conditions as described in Materials and Methods, or we measured the total pixel intensity of the immunostaining for individual proteins. We found that both methods gave essentially the same result. However, we found that pixel intensity measurements displayed much greater variance than the quantitation of area. Hence, all immunofluorescence quantitation is presented as area percentage of control.

NrCAM in the PNS and brevican in the CNS were completely absent from nodes at 8 weeks (data not shown) and 16 weeks post-tamoxifen (Fig. $2 A, B$ ). A third protein, gliomedin, which has also been proposed to interact directly with Nfasc186, was retained at high levels ( $~ 80 \%$ of control) up to 8 weeks but then declined to $20 \%$ of the control value by 16 weeks posttamoxifen (Eshed et al., 2005; Fig. 2A,C). The delay in the loss of gliomedin may reflect its supplementary interactions with extracellular matrix at the PNS node (Eshed et al., 2007).

In contrast to gliomedin in the PNS, the amounts of $\beta 1$ subunit of the sodium channel $(\beta 1 \mathrm{Nav})$, ankyrin $\mathrm{G}$, and $\beta \mathrm{IV}$ spectrin were commensurate with those of Nav, and sodium channels themselves reached a level that did not change significantly from 8 to 16 weeks, namely between 65 and $70 \%$ of control values (Fig. $2 A, C)$. These data suggested that a complex comprising Nav/ $\beta 1 N a v / a n k y r i n$ G/ $\beta I V$ spectrin remained clustered at the PNS node despite the almost complete absence of Nfasc186, although, of course, paranodes were still intact. Despite this limited resistance to disruption, the function of quadriceps nerves was significantly compromised. Conduction velocities were reduced by $38 \%$ in TCE/Nfasc $\mathrm{C}^{\mathrm{fl}-}$ compared with $\mathrm{Nfasc}^{\mathrm{fl} / \mathrm{-}}$ mice 16 weeks post-tamoxifen from $45.6 \pm 3.5$ to $28.1 \pm$ $2.0 \mathrm{~ms}^{-1}$ (mean \pm SEM; $p=0.0024 ; n=5$ per condition).

In the CNS we observed much more severe effects on the accessory protein $\beta 1 \mathrm{Nav}$ and the cytoskeletal linker proteins ankyrin $\mathrm{G}$ and $\beta$ IV spectrin. When $<6 \%$ of nodal Nfasc186 remained by 8 weeks post-tamoxifen, there were substantial reductions in the amounts of $\beta 1 \mathrm{Nav}$ and ankyrin G. In contrast, $\beta \mathrm{IV}$ spectrin and Nav were much less affected ( $>80 \%$ of control) (Fig. $2 B, D$ ). After another 8 weeks there were no further reductions in $\beta 1 \mathrm{Nav}$ and ankyrin $\mathrm{G}$, but $\beta \mathrm{IV}$ spectrin at the node declined to levels that were now commensurate with these proteins (Fig. $2 B, D$ ). Surprisingly, Nav were less susceptible to disruption (Fig. $2 B, D$ ).

These data point to the differential effects of reducing neuronal neurofascin at the node of Ranvier in the CNS versus the PNS, which implies different mechanisms of stabilizing the nodal 
complex at these two locations. A further difference in the consequences of withdrawing neuronal neurofascin from the node was the collateral effect on CNS paranodes, which was not observed in the PNS. The localization of glial neurofascin at the paranodes was extensively disrupted in the mutant by 16 weeks after tamoxifen treatment, although it can also be observed that staining in the CNS control was also not uniformly intact (Fig. $2 B$ ). To study this issue further in the CNS, we asked whether such apparent paranodal disruption might influence Nav retention at the node.

Given the more extensive disruption of the CNS compared with the PNS node, plus the significant reduction in PNS conduction velocity, it was perhaps not surprising that the mice died within 2 weeks of the last time point (16 weeks posttamoxifen). This prevented us from determining whether further disruption of the PNS nodal complex followed the path of the CNS node.

\section{Loss of neuronal neurofascin disrupts paranodes}

Representative images of the distribution of Nav, Caspr, and Kv1.1 channels in control and mutant teased fibers from the spinal cord revealed both intact and disrupted paranodes (Fig. 3A). Disruption in the mutant was not a consequence of losing the transmembrane components of the junction since we had already shown that the amount of the glial Nfasc 155 was unaffected in the mutant (Fig. 1A), and the other two neuronal components of this cell surface adhesion complex, Caspr and contactin, were similarly unaffected (Fig. 3B). To determine whether restriction of Nav to the node is influenced by the intactness of paranodal protein localization both in controls and when the levels of neuronal neurofascin are severely reduced, the mean Nav area was quantitated either when Kv channels were completely segregated to the juxtaparanodes (intact paranodes) or when $\mathrm{Kv}$ immunoreactivity invaded the paranode (disrupted paranodes).

Disrupted paranodes were observed in both control and mutant nerves (data not shown). Hence, we first determined whether control mice ( $N f a s c^{f l-}$ ) were significantly different from wild-type mice $\left(\mathrm{Nfasc}^{+/+}\right)$, and they were not (Fig. 3C).

However, when the CNS nodal complex was deranged in mutant mice, there was an almost threefold increase in the number of disrupted paranodes compared with control mice (Nfasc $\mathrm{fl}^{\mathrm{fl}-}$; Fig. $3 \mathrm{C}$ ). Nevertheless, this deranged paranodal protein localization did not affect Nav retention in either control or mutant mice (Fig. 3D).
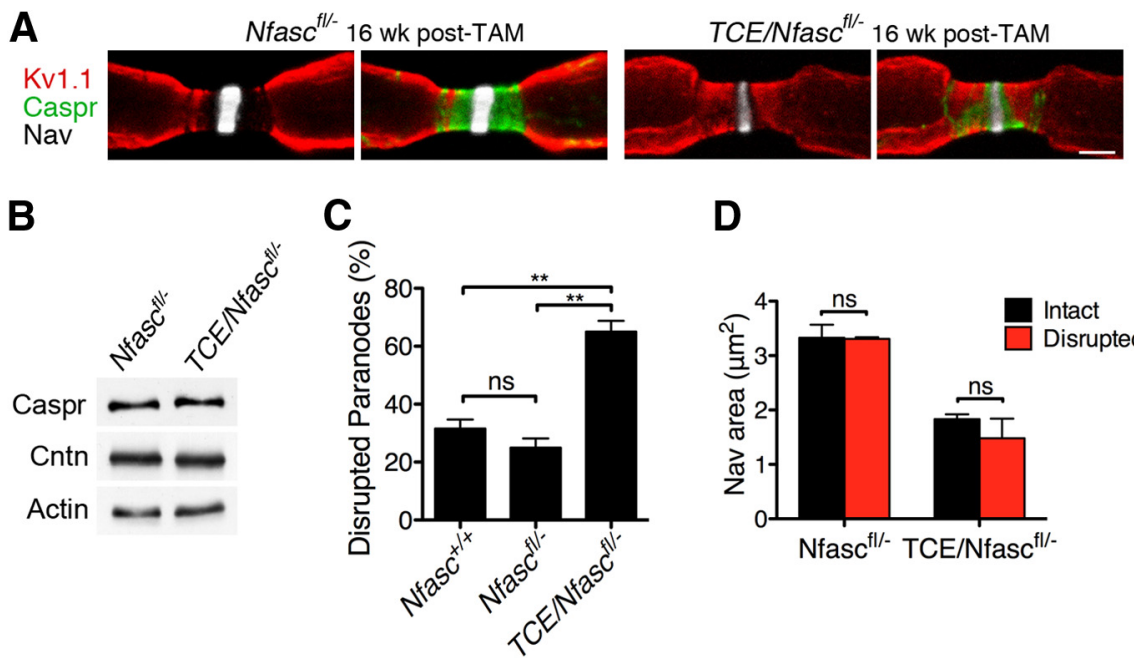

D

Figure 3. CNS paranodal and juxtaparanodal proteins are mislocalized following Nfasc 186 deletion. $A$, Immunofluorescence of teased ventral funiculi from Nfasc $\mathrm{fl}^{-}$and TCE/Nfast $\mathrm{fl}^{f /-}$ mice 16 weeks (wk) post-tamoxifen treatment shows disrupted Caspr staining and relocalization of voltage-gated potassium channel (Kv) at the paranodes of mutants. Scale bar, $3 \mu \mathrm{m}$. $\boldsymbol{B}$, Western blotting of spinal cord lysates from control and mutant mice 16 weeks post-tamoxifen shows no reduction in Caspr or contactin in mutant nerves. Actin was the loading control. C, The percentage of paranodes displaying invasive Kv immunoreactivity was measured in wild-type $\left(\mathrm{Nfasc}^{+/+}\right)$, control $\left(\mathrm{NfasC}^{f /-}\right)$, and mutant $\left(\mathrm{TCE}_{\mathrm{NfasC}} \mathrm{fl}^{-}\right.$) mice 16 weeks post-tamoxifen (wild-type mice were the same age but not treated with tamoxifen) and are displayed as means \pm SEM. There is a significant increase in disrupted paranodes in mutants compared with controls (one-way ANOVA followed by Tukey's multiple-comparison test; ${ }^{* *} p=$ $0.0001, n=3,120$ paranodes per condition). ns, not significant. $\boldsymbol{D}$, The mean areas of Nav immunoreactivity were measured when either paranodes were intact (Kv restricted to juxtaparanodes) or disrupted (Kv invasion of paranodes). In the control and the mutant, there is no significant difference (ns) in Nav localization at the nodes when paranodes are either intact or disrupted (mean \pm SEM, one-way ANOVA followed by Tukey's multiple-comparison tests; $p=0.01, n=3$, minimum 90 nodes).
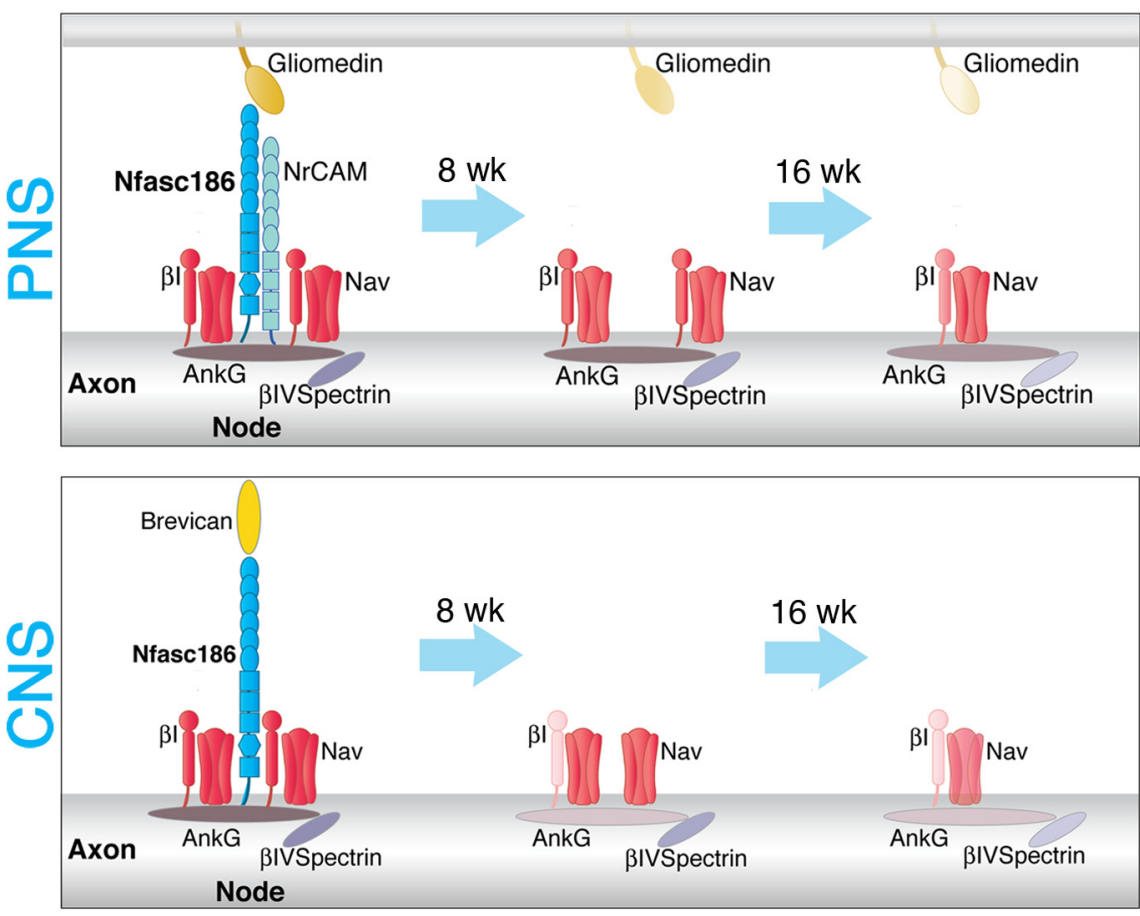

Figure 4. Diagram contrasting the sequence of events when Nfasc186 is lost in the PNS and CNS. The intensity of the colors indicates when proteins are diminished but not completely lost.

\section{Discussion}

The complete absence of NrCAM and brevican at PNS and CNS nodes, respectively, when depleted of Nfasc186 is consistent with considerable evidence for the direct interaction of Nfasc186 with these proteins (Hedstrom et al., 2008; Zonta et al., 2011). The loss 
of $80 \%$ of gliomedin at PNS nodes was also consistent with previous observations on its interactions with Nfasc186 (Eshed et al., 2005), although residual gliomedin may reflect its ability to also interact with extracellular matrix proteins (Eshed et al., 2007). Similarly, although $\beta 1 \mathrm{Nav}$ is known to interact with neuronal neurofascin (McEwen and Isom, 2004) in the PNS, this is not required to maintain it at the node, although reduced amounts would be predicted to affect the function of the channel, as we observed (Isom, 2002).

The fact that a complex comprising Nav/ $\beta 1$ Nav/ankyrin G/ $\beta$ IV spectrin remained largely clustered at the PNS node despite the almost complete absence of Nfasc186 suggested that intact paranodal axoglial junctions in the PNS restricted diffusion of this complex away from the node. Certainly, when paranodal axoglial junctions are completely disrupted, nodal components diffuse away (Rios et al., 2003; Pillai et al., 2009). In the PNS, where the nodal complex remained relatively intact, there was a substantial 38\% reduction in the speed of nerve conduction. It is perhaps surprising that a $35 \%$ loss in Nav area should lead to such a significant reduction. However, this was accompanied by a $52 \%$ loss in $\beta 1 \mathrm{Nav}$, and this subunit is known to be an essential modulator of sodium channel function at nodes of Ranvier (Chen et al., 2004). Furthermore, the measured reductions of nodal components are based on area alone and may underestimate the overall loss in Nav, since there was also a reduction of pixel intensity per area (data not shown).

The more rapid loss of Nfasc186 after tamoxifen treatment in the CNS suggests that nodal Nfasc186 may be more stable at PNS nodes. Moreover, this greater loss in the CNS was accompanied by major reductions in the nodal content of $\beta 1 \mathrm{Nav}$ and ankyrin $\mathrm{G}$, even though Nav and $\beta \mathrm{IV}$ spectrin levels remained high ( $>80 \%$ of control). This suggests that in the CNS, interaction of Nfasc 186 with $\beta 1 \mathrm{Nav}$ and ankyrin $\mathrm{G}$ plays a more important role in stabilizing these proteins at the CNS node than in the PNS. Nevertheless, by 16 weeks post-tamoxifen, $\beta$ IV spectrin in the mutant was reduced to levels commensurate with those of $\beta 1 \mathrm{Nav}$ and ankyrin $\mathrm{G}$, indicating that interaction of $\beta \mathrm{IV}$ spectrin with these former proteins, and most likely ankyrin $G$, is necessary to stabilize $\beta$ IV spectrin at the node. Surprisingly, sodium channels were still retained to a large degree even at 16 weeks posttamoxifen ( $\sim 60 \%$ of control). Since brevican was completely lost, it seems likely that paranodal axoglial junctions have a particularly important role in retaining Nav at the node, although the possible involvement of other $\beta$ subunits of the sodium channel cannot be ruled out. Figure 4 is a diagram that summarizes the contrasting sequence of events in the PNS and CNS upon the loss of Nfasc186.

Age-related paranodal disruption has been reported in the CNS (Hinman et al., 2006). However, our data indicate that this does not exacerbate Nav loss at nodes lacking Nfasc186, at least in the time frame of these experiments. Nevertheless, it is of considerable interest that loss of neuronal neurofascin amplifies paranodal disruption. The precise mechanism by which this is achieved is not clear, but it seems likely to involve the axonal cytoskeleton since both ankyrin $\mathrm{G}$ and $\beta \mathrm{IV}$ spectrin are highly depleted at CNS nodes in the mutant. Disruption of paranodes due to loss of Nfasc186 during development has also been noted by others (Thaxton et al., 2011). In the latter study, disruption of paranodes was observed in both PNS and CNS. Similarly, in a companion article disruption of PNS paranodes was induced by the complete absence of gliomedin and NrCAM in peripheral nerves during development and adulthood (Amor et al., 2014). We only observed this phenomenon in the CNS, which may reflect the fact we studied ablation in adult mice, where the relative stability of the preformed Nav/ $\beta 1 \mathrm{Nav} /$ ankyrin G/ $\beta \mathrm{IV}$ spectrin complex at the PNS node retards or may even prevent further disturbance to the adjacent sub-paranodal axonal cytoskeleton altogether.

Given that many of the core components of the nodal complex are identical in the CNS and PNS, it was perhaps surprising that loss of neuronal neurofascin should differentially affect the stability of the nodal complexes in the PNS versus the CNS, and this difference was particularly pronounced for the cytoskeletalassociated protein ankyrin $\mathrm{G}$, which is known to interact directly with Nfasc186 (Garver et al., 1997). Nevertheless, the fact that the retention of sodium channels in the CNS was comparable in the CNS and PNS does suggest that the axoglial junctions may have a particularly important function in maintaining Nav at the CNS node. It may also be the case that other ankyrin isoforms, known to be expressed in CNS neurons, compensate for the loss of ankyrin G (Kordeli and Bennett, 1991). This differential susceptibility to disruption may underpin the exacerbation of CNS axonal damage due to Nfasc186-specific antibodies in experimental allergic encephalomyelitis, an animal model of multiple sclerosis (Lindner et al., 2013).

\section{References}

Amor V, Feinberg K, Eshed-Eisenbach Y, Vainshtein A, Frechter S, Grumet M, Rosenbluth J, Peles E (2014) Long-term maintenance of $\mathrm{Na}^{+}$channels at nodes of Ranvier depends on glial contact mediated by gliomedin and NrCAM. J Neurosci 34:5089-5098.

Bhat MA, Rios JC, Lu Y, Garcia-Fresco GP, Ching W, St Martin M, Li J, Einheber S, Chesler M, Rosenbluth J, Salzer JL, Bellen HJ (2001) Axonglia interactions and the domain organization of myelinated axons requires neurexin IV/Caspr/Paranodin. Neuron 30:369-383. CrossRef Medline

Boyle ME, Berglund EO, Murai KK, Weber L, Peles E, Ranscht B (2001) Contactin orchestrates assembly of the septate-like junctions at the paranode in myelinated peripheral nerve. Neuron 30:385-397. CrossRef Medline

Charles P, Tait S, Faivre-Sarrailh C, Barbin G, Gunn-Moore F, DenisenkoNehrbass N, Guennoc AM, Girault JA, Brophy PJ, Lubetzki C (2002) Neurofascin is a glial receptor for the paranodin/Caspr-contactin axonal complex at the axoglial junction. Curr Biol 12:217-220. CrossRef Medline

Chen C, Westenbroek RE, Xu X, Edwards CA, Sorenson DR, Chen Y, McEwen DP, O'Malley HA, Bharucha V, Meadows LS, Knudsen GA, Vilaythong A, Noebels JL, Saunders TL, Scheuer T, Shrager P, Catterall WA, Isom LL (2004) Mice lacking sodium channel betal subunits display defects in neuronal excitability, sodium channel expression, and nodal architecture. J Neurosci 24:4030-4042. CrossRef Medline

Court FA, Sherman DL, Pratt T, Garry EM, Ribchester RR, Cottrell DF, Fleetwood-Walker SM, Brophy PJ (2004) Restricted growth of Schwann cells lacking Cajal bands slows conduction in myelinated nerves. Nature 431:191-195. CrossRef Medline

Davis JQ, Lambert S, Bennett V (1996) Molecular composition of the node of Ranvier: identification of ankyrin-binding cell adhesion molecules neurofascin (mucin+/third FNIII domain-) and NrCAM at nodal axon segments. J Cell Biol 135:1355-1367. CrossRef Medline

Eshed Y, Feinberg K, Poliak S, Sabanay H, Sarig-Nadir O, Spiegel I, Bermingham JR Jr, Peles E (2005) Gliomedin mediates Schwann cell-axon interaction and the molecular assembly of the nodes of Ranvier. Neuron 47: 215-229. CrossRef Medline

Eshed Y, Feinberg K, Carey DJ, Peles E (2007) Secreted gliomedin is a perinodal matrix component of peripheral nerves. J Cell Biol 177:551-562. CrossRef Medline

Feinberg K, Eshed-Eisenbach Y, Frechter S, Amor V, Salomon D, Sabanay H, Dupree JL, Grumet M, Brophy PJ, Shrager P, Peles E (2010) A glial signal consisting of gliomedin and NrCAM clusters axonal $\mathrm{Na}+$ channels during the formation of nodes of Ranvier. Neuron 65:490-502. CrossRef Medline

Garver TD, Ren Q, Tuvia S, Bennett V (1997) Tyrosine phosphorylation at a site highly conserved in the L1 family of cell adhesion molecules abolishes 
ankyrin binding and increases lateral mobility of neurofascin. J Cell Biol 137:703-714. CrossRef Medline

Hedstrom KL, Ogawa Y, Rasband MN (2008) AnkyrinG is required for maintenance of the axon initial segment and neuronal polarity. J Cell Biol 183:635-640. CrossRef Medline

Hinman JD, Peters A, Cabral H, Rosene DL, Hollander W, Rasband MN, Abraham CR (2006) Age-related molecular reorganization at the node of Ranvier. J Comp Neurol 495:351-362. CrossRef Medline

Isom LL (2002) Beta subunits: players in neuronal hyperexcitability? Novartis Found Symp 241:124-138; discussion 138-143, 226-132. Medline

Jenkins SM, Bennett V (2002) Developing nodes of Ranvier are defined by ankyrin-G clustering and are independent of paranodal axoglial adhesion. Proc Natl Acad Sci U S A 99:2303-2308. CrossRef Medline

Kordeli E, Bennett V (1991) Distinct ankyrin isoforms at neuron cell bodies and nodes of Ranvier resolved using erythrocyte ankyrin-deficient mice. J Cell Biol 114:1243-1259. CrossRef Medline

Lindner M, Ng JK, Hochmeister S, Meinl E, Linington C (2013) Neurofascin 186 specific autoantibodies induce axonal injury and exacerbate disease severity in experimental autoimmune encephalomyelitis. Exp Neurol 247:259-266. CrossRef Medline

McEwen DP, Isom LL (2004) Heterophilic interactions of sodium channel betal subunits with axonal and glial cell adhesion molecules. J Biol Chem 279:52744-52752. CrossRef Medline

Menegoz M, Gaspar P, Le Bert M, Galvez T, Burgaya F, Palfrey C, Ezan P, Arnos F, Girault JA (1997) Paranodin, a glycoprotein of neuronal paranodal membranes. Neuron 19:319-331. CrossRef Medline

Pillai AM, Thaxton C, Pribisko AL, Cheng JG, Dupree JL, Bhat MA (2009) Spatiotemporal ablation of myelinating glia-specific neurofascin (Nfasc NF155) in mice reveals gradual loss of paranodal axoglial junctions and concomitant disorganization of axonal domains. J Neurosci Res 87:17731793. CrossRef Medline

Rios JC, Rubin M, St Martin M, Downey RT, Einheber S, Rosenbluth J, Levinson SR, Bhat M, Salzer JL (2003) Paranodal interactions regulate expression of sodium channel subtypes and provide a diffusion barrier for the node of Ranvier. J Neurosci 23:7001-7011. Medline

Sherman DL, Tait S, Melrose S, Johnson R, Zonta B, Court FA, Macklin WB, Meek S, Smith AJ, Cottrell DF, Brophy PJ (2005) Neurofascins are required to establish axonal domains for saltatory conduction. Neuron 48: 737-742. CrossRef Medline

Sherman DL, Krols M, Wu LM, Grove M, Nave KA, Gangloff YG, Brophy PJ (2012) Arrest of myelination and reduced axon growth when Schwann cells lack mTOR. J Neurosci 32:1817-1825. CrossRef Medline

Tait S, Gunn-Moore F, Collinson JM, Huang J, Lubetzki C, Pedraza L, Sherman DL, Colman DR, Brophy PJ (2000) An oligodendrocyte cell adhesion molecule at the site of assembly of the paranodal axo-glial junction. J Cell Biol 150:657-666. CrossRef Medline

Thaxton C, Pillai AM, Pribisko AL, Dupree JL, Bhat MA (2011) Nodes of Ranvier act as barriers to restrict invasion of flanking paranodal domains in myelinated axons. Neuron 69:244-257. CrossRef Medline

Yang Y, Lacas-Gervais S, Morest DK, Solimena M, Rasband MN (2004) BetaIV spectrins are essential for membrane stability and the molecular organization of nodes of Ranvier. J Neurosci 24:9230-9240. Medline

Zonta B, Tait S, Melrose S, Anderson H, Harroch S, Higginson J, Sherman DL, Brophy PJ (2008) Glial and neuronal isoforms of Neurofascin have distinct roles in the assembly of nodes of Ranvier in the central nervous system. J Cell Biol 181:1169-1177. CrossRef Medline

Zonta B, Desmazieres A, Rinaldi A, Tait S, Sherman DL, Nolan MF, Brophy PJ (2011) A critical role for Neurofascin in regulating action potential initiation through maintenance of the axon initial segment. Neuron 69:945956. CrossRef Medline 